many challenges. This study was undertaken to evaluate the feasibility of implementing a point-of-care p24 antigen detection test (LYNX) in rural Zambia.

Methods A cross-sectional study of infants attending the Macha Hospital HIV or primary care clinics for early infant diagnosis was conducted in Choma district, Southern Province, Zambia during 2014 and 2015. Two blood samples were collected from each participant, one for immediate testing with the LYNX test and a second for standard HIV DNA testing at a central laboratory. Counsellors were trained to perform the LYNX test and observed for adherence to protocols.

Results A total of 210 LYNX tests were performed; 93\% of tests were run according to protocol with a result available with a median time of 55 minutes (IQR:54, 57); 10\% of tests were run on battery power. The median turnaround time for the availability of the HIV DNA test result was 2.5 months (IQR: $1.8,5.0)$. The sensitivity and specificity of the LYNX test were $70 \%$ and $100 \%$, respectively. Challenges to implementation included the long duration of the LYNX test and multiple steps, disruption of other daily activities, and managing variable patient volumes.

Conclusions Point-of-care tests for early infant diagnosis are urgently needed to increase access to testing. The LYNX test was successfully performed by counsellors and had several characteristics facilitating implementation in rural clinics. The LYNX test could address many challenges to testing in rural areas and allow for earlier diagnosis and treatment of HIV-infected infants, therefore improving outcome.

\section{PA-097 FEASIBILITY OF USING THE LYNX POINT-OF-CARE TEST FOR EARLY INFANT HIV DIAGNOSIS IN RURAL ZAMBIA}

Mutinta Chilikwazi, Catherine Sutcliffe, Philip Thuma, Kathy Sinywimaanzi, Hellen Matakala, Passwell Munachoonga, William Moss. Macha Research Trust, Zambia

10.1136/bmjgh-2016-000260.128

Background Early infant diagnosis of HIV is challenging in sub-Saharan Africa, particularly in rural areas, leading to delays in diagnosis and treatment. A point-of-care test would overcome 